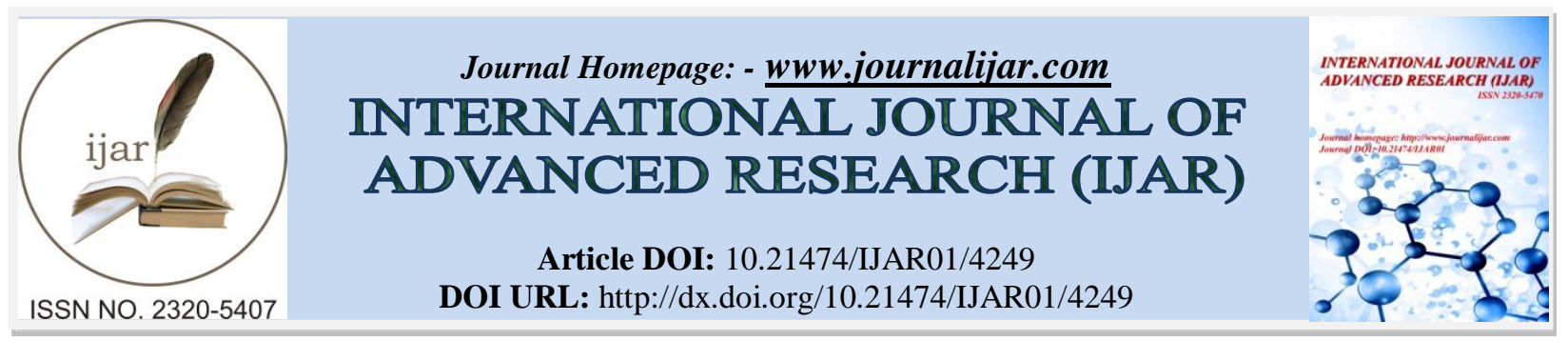

RESEARCH ARTICLE

\title{
INHIBITION OF ISPD ENZYME TO CONTROL THE GROWTH OF MYCOBACTERIUM TUBERCULOSIS.
}

\author{
Manoj Kumar ${ }^{1}$, Pratik Kumar ${ }^{2}$, Pratima Kumari ${ }^{1}$ and Jainendra Kumar ${ }^{3}$. \\ 1. Post Graduate Centre \& Department of Botany and Biotechnology, College of Commerce, Arts \& Science, \\ Patna (Bihar), India 800020. \\ 2. Intermediate Reference Laboratory, TBDC Campus, Agamkuan, Patna, Bihar 800007. \\ 3. Ashayan, D.P.R. Colony, Ramjaipal Road, Off West Bailey Road, P.O. Danapur (Bihar) Patna 801503.
}

\section{Manuscript Info}

(........................

Manuscript History

Received: 25 March 2017

Final Accepted: 27 April 2017

Published: May 2017

Key words:-

IspD (CDP-ME Cytidylyltransferase),

Rosuvastatin, Mycobacterium

tuberculosis, molecular docking,

SwissDock

\section{Abstract}

Like several other pathogens, Mycobacterium tuberculosis synthesizes isopentenyl diphosphate via the 2-C-methyl-D-erythritol 4-phosphate (MEP) pathway. IspD (2C-methyl-D-erythritol-4-phosphate Cytidyltransferase) is a flexible enzyme that carries out the third step in MEP pathway where 2-C-methyl-D-erythritol 4 phosphate (MEP) condenses with Cytidine Triphosphate (CTP) to form 4diphosphocytidyl-2-amethyl-D-erythritol (CDP-ME). It catalizes the transfer of a cytidyl group from CTP to methylerythritol phosphate and utilizes two substrates, MEP and CTP. Due to this feature, this stage of MEP pathway is a significant reaction step that can be targeted to control the spread of the organism in vivo. Rosuvastatin successfully docks as a ligand close to the active site of the IspD with involvement of low (negative) energy indicating a stable system and a likely binding interaction. It can, thus, be safely assumed that rosuvastatin molecule could possibly be used as a candidate to competitively inhibit IspD to bind to CTP blocking the MEP pathway and keep the proliferating Mycobacterium tuberculosis under control.

Copy Right, IJAR, 2017,. All rights reserved.

\section{Introduction:-}

Tuberculosis (TB), caused by the bacterial pathogen Mycobacterium tuberculosis is one of the oldest recorded human ailments and one of the biggest killers among the infectious diseases (Smith, 2003). New vaccines and drugs are needed to control its worldwide epidemic which assumingly kills two million people each year. To develop new anti-tubercular drug candidates, it is essential to understand the genetics and physiology of M. tuberculosis along with its interaction with the host. Search for new molecules that can check the growth and spread of the organism at some early stage of its development is the need of the time. It is urgently required that organism's metabolic targets are identified for novel therapeutics (Brown et al., 2010).

Currently used antibiotics are mostly known to target cell wall biosynthesis in the bacterium. Drugs like isoniazid and ethionamide target the synthesis of mycolic acids and those as ethambutol target arabinogalactan and lipoarabinomannan. A good target might be an enzyme of the Isoprenoid biosynthesis that the organism essentially requires to produce several cellular components including those involved in cell wall formation.

Corresponding Author:- Manoj Kumar.

Address:- Post Graduate Centre \& Department of Botany and Biotechnology College of Commerce, Arts 
M. tuberculosis synthesizes the isoprenoid precursor isopentenyl diphosphate (IPP) via 1-deoxy-D-xylulose 5phosphate (DOXP) pathway (non-mevalonate pathway). Targeting an enzyme of MEP pathway would leave the human mevalonate pathway alone sparing us from the side effects of the drug.

The non-mevaslonate pathway starts with condensation of pyruvate and glyceraldehyde-3-phosphate which yields 1deoxy-D-xylulose 5-phosphate (DOXP). DOXP is converted to 2-C-methyl-D-erythritol 4-phosphate (MEP) which after condensation with CTP forms 4-diphosphocytidyl-2-amethyl-D-erythritol (CDPME) in stage III. Then CDPME is phosphorylated to 4-diphosphocytidyl-2-amethyl-D-erythritol 2-phosphate (CDPME2P) and to 2-C-methyl-Derythritol-2,4 cyclodiphosphate (ME2,4cPP) which is the last known intermediate of the DOXP/MEP pathway (Wanke et al., 2001).

Stage III of the pathway is executed by the enzyme 4-Diphosphocytidyl-2C-methyl-D-erythritol Cytidyltransferase (IspD) which is a homodimer. Each subunit of the dimer is a single domain $\alpha / \beta$ structure constructed around a seven-stranded twisted $\beta$-sheet into which is inserted an extended " $\beta$-arm." Two arms associate to form the dimer interface that involves numerous hydrogen bonds and salt bridges (Fig. 1).

The active site is created at the dimer interface by seven polypeptide segments, six from one subunit and one from the partner (Hunter, 2007).

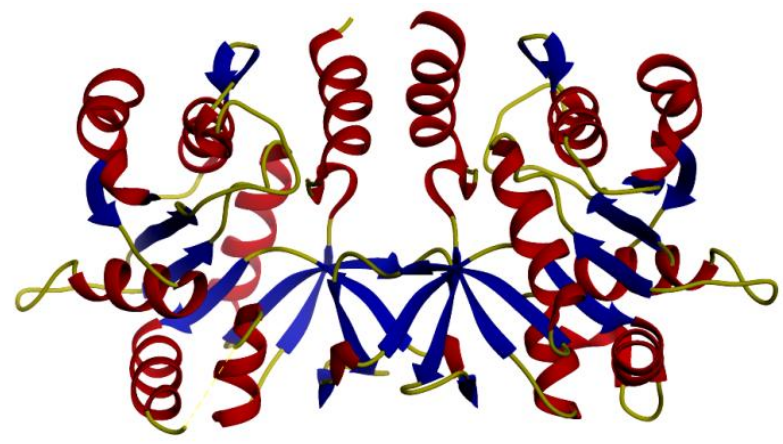

Fig.1:- 3D structure of the dimer IspD.

\section{Materials and Methods:-}

3D structural coordinates of 2-C-methyl-D-erythritol 4-phosphate cytidyltransferase (IspD) of Mycobacterium tuberculosis complexed with CTP was downloaded from www.rcsb.org/pdb with the assigned pdb code 3Q7U (Reddy et al., 2011). UCSF Chimera (Pettersen et al., 2004) was used to model the structure, remove a chain of the dimer along with the CTP and prepare Chain A for docking with rosuvastatin. 3D coordinates of rosuvastatin (SDF file) was downloaded from PubChem (CID: 446157) and converted to Mol2 file by UCSF Chimera. Docking was set online on SwissDock, a protein-small molecule docking web service based on EADock DSS (Grosdidier et al., 2011).

\section{Results and Discussion:-}

The calculations in SwissDock are performed in the CHARMM force field. When using default parameters, the whole target protein structure is considered during the docking. The performance of EADock DSS depends on the number of free dihedral angles of the ligand (Grosdidier et al., 2011). The Rosuvastatin $\left(\mathrm{C}_{22} \mathrm{H}_{28} \mathrm{FN}_{3} \mathrm{O}_{6} \mathrm{~S}\right)$ comprises 10 rotatable bond count having molecular weight $481.539 \mathrm{~g} / \mathrm{mol}$ with 3 hydrogen bond donor count and 10 hydrogen bond acceptor count. EADock DSS's algorithm generates upto 15,000 binding modes in the vicinity of the target cavities of the protein surface (blind docking) estimating their CHARMM energies on a grid. Binding Modes (BMs) with the most favorable energies are ranked, taking account of the solvent effect using the FACTS implicit solvation model, and clustered. Finally, the most favorable clusters are presented into the result file (Grosdidier et al., 2011). 


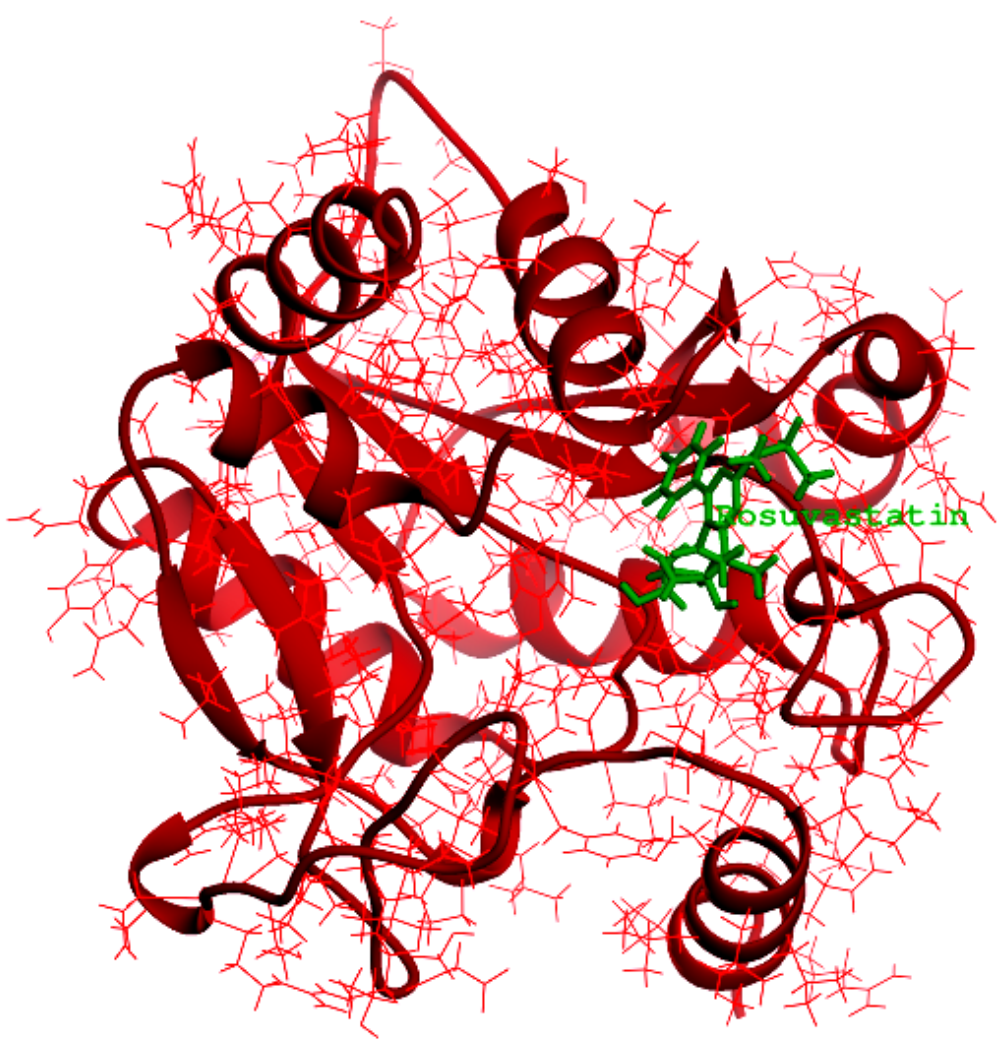

Fig. 2:- Chain A of IspD docked with ligand (Rosuvastatin) Cluster 19 (Cluster rank 1).

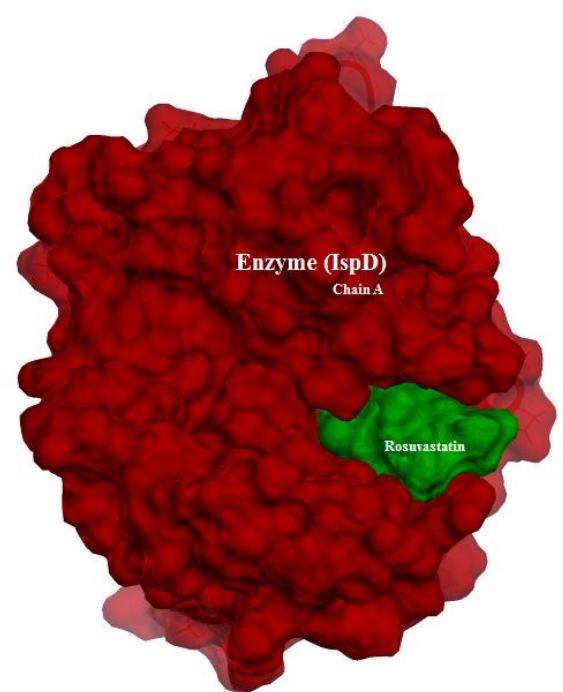

Fig. 3:- Surface view of the ligand docked into the IspD at lowest energy. 


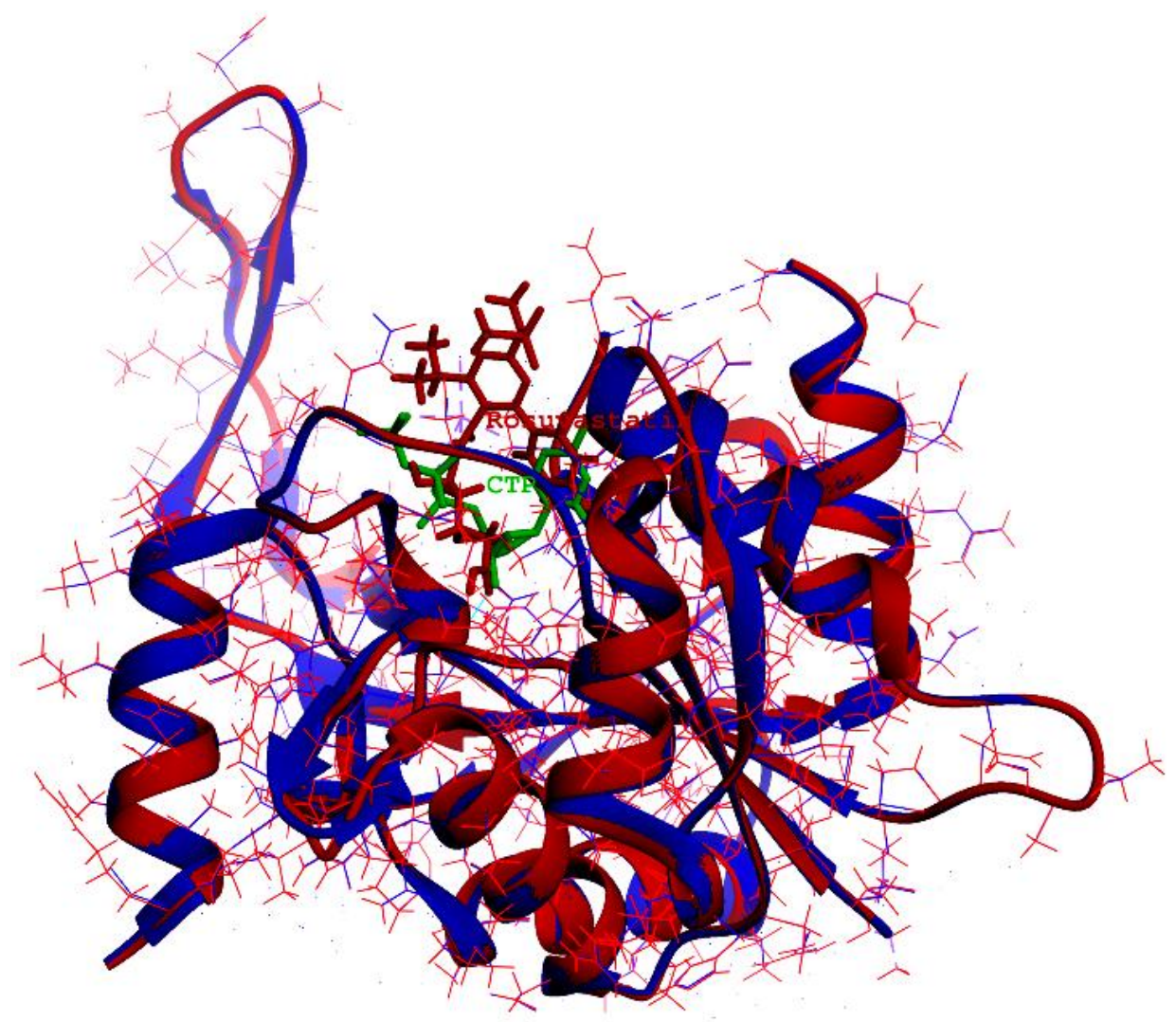

Fig. 4:- Chain A (red) of IspD with the ligand superposed with the crystal structure 3Q7U Chain A (blue) complexed with CTP

Cluster 19 of SwissDock output ranked 1 predicted the best docking mode/binding mode with Energy 0.158562, SimpleFitness -0.158562, FullFitness -1413.9502, InterFull -57.3238, IntraFull -66.3874, solvFull 1463.47, surfFull 173.231, extraFull 0.0, deltaGcompsolvpol -1463.47, deltaGcompsolvnonpol 173.231, deltaGprotsolvpol -1491.03, deltaGprotsolvnonpol 174.309, deltaGligsolvpol -21.7472, deltaGligsolvnonpol 10.994, deltaGvdw -57.3238, deltaGelec 0.0 and deltaG -7.537239. Figs. 2 and 3 depict the rendered structure of the best ligand pose in ribbon and surface views respectively. Fig. 4 presents the superposition of the crystal structure of Chain A of IspD complexed with the CTP (Reddy et al., 2011). Björkelid et al. (2011) had established the existence of potential flexibility in areas close to the active site in mycobacterial IspD..

The best binding mode of rosuvastatin with the Chain A of the enzyme is almost exactly where CTP binds. It helps to assume that rosuvastatin would bind more reliably and prevent the CTP from binding at the dimer interface where chain A contributes six polypeptide segments. The observation, however, needs support from in vitro tests. Varikoti et al. (2012) attempted structure based de novo designed possible inhibitor molecules for docking into the active pocket of IspD enzyme. Docking of the top hit virtual compound with docking score of -7.559 into the active site showed binding to the hinge region by forming H-bonds with the peptide backbone of Arg20 and Lys215. ObiolPardo et al. (2010) modelled IspD of Mycobacterium tuberculosis to derive a reliable model for use in structure based drug design and they proposed that electrostatic interactions might result in higher affinity of a ligand in comparison of the CTP.

In case of Plasmodium, Price et al. (2016) could identify 2-phenyl benzo[d]isothiazol-3(2H)-ones as speciesselective inhibitors of IspD. In culture, these compounds displayed nanomolar inhibitory activity preventing the growth of P. falciparum. Imlay et al. (2015) found that Plasmodium IspD could also be an easy intracellular target of MMV008138. 


\section{References:-}

1. Björkelid, C., Bergfors, T., Henriksson, L.M., Stern, A.L., Unge, T., Mowbray, S.L. and Jones, T.A. 2011 Structural and functional studies of mycobacterial IspD enzymes. Acta Crystallogr D Biol Crystallogr. 67(5):40314. DOI: $10.1107 /$ S0907444911006160.

2. Brown, A.C., Eberl, M., Crick, D.C., Jomaa, H. and Parish, T. 2010 The Nonmevalonate Pathway of Isoprenoid Biosynthesis in Mycobacterium tuberculosis Is Essential and Transcriptionally Regulated by Dxs. Journal of Bacteriology 192(9):2424-2433. DOI:10.1128/JB.01402-09

3. Grosdidier, A., Zoete, V. and Michielin, O. 2011 SwissDock, a protein-small molecule docking web service based on EADock DSS. Nucleic Acids Res. 39(Web Server issue):W270-7. DOI: 10.1093/nar/gkr366.

4. Hunter, W.N. 2007 The Non-mevalonate Pathway of Isoprenoid Precursor Biosynthesis. The Journal of Biological Chemistry 282:21573-21577. DOIi: 10.1074/jbc.R700005200

5. Imlay, L.S., Armstrong, C.M., Masters, M.C., Li, T., Price. K.E., Edwards, R.L., Mann, K.M., Li, L.X., Stallings, C.L., Berry, N.G., O’Neill, P.M. and Audrey R. Odom, A.R. 2015 Plasmodium IspD (2-C-Methyl-Derythritol 4-Phosphate Cytidyltransferase), an Essential and Druggable Antimalarial Target. ACS Infect Dis.; 1(4): 157-167. DOI: $10.1021 /$ id500047s.

6. Obiol-Pardo, C., Cordero, A., RubioMartinez, J. and Imperial, S. (2010) Homology modeling of Mycobacterium tuberculosis 2CmethylDerythritol4phosphate cytidylyltransferase, the third enzyme in the MEP pathway for isoprenoid biosynthesis. J Mol Model. 16(6):106173. DOI: 10.1007/s008940090615x.

7. Pettersen, E.F., Goddard, T.D., Huang, C.C., Couch, G.S., Greenblatt, D.M., Meng, E.C. and Ferrin, T.E. 2004 UCSF Chimera--a visualization system for exploratory research and analysis. .J Comput Chem. 25(13):1605-12.

8. Price, K.E., Armstrong, C.M., Imlay, L.S., Hodge, D.M., Pidathala, C., Roberts, N.J., Park, J., Makati, M., Raman Sharma, R., Alexandre S. Lawrenson, A.S., Tolia, N.H., Berry, N.G., Paul M. O'Neill, P.M. and John, A.R.O. 2016 Molecular Mechanism of Action of Antimalarial Benzoisothiazolones: Species-Selective Inhibitors of the Plasmodium spp. MEP Pathway enzyme, IspD. Scientific Reports 6, Article number: 36777. DOI:10.1038/srep36777.

9. Reddy, M.C.M, Bruning, J.B., Thurman, C, Ioerger, T.R. and Sacchettini, J.C. 2011 Crystal structure of Mycobacterium tuberculosis 2-C-methyl-D-erythritol 4-phosphate cytidyltransferase (IspD): a candidate antitubercular drug target. www.rcsb.org, Proteins. DOI: 102210/pdb2q7u/pdb.

10. Smith, I. 2003 Mycobacterium tuberculosis Pathogenesis and Molecular Determinants of Virulence. Clinical Microbiology Reviews 16(3):463-496. DOI: 10.1128/CMR.16.3.463-496.

11. Varikoti, R.A., Gangwal, R.P., Dhoke, G.V., Venkata Krishnan, R. and Abhay T. Sangamwar, A.T. 2012 Structure based de novo design of IspD inhibitors as anti-tubercular agents. Available from Nature Precedings http://dx.doi.org/10.1038/npre.2012.7088.1

12. Wanke, M., Skorupinska-Tudek, K. and Swiezewska, E. 2001 Isoprenoid biosynthesis via 1-deoxy-D-xylulose 5-phosphate/2-C-methyl-D-erythritol 4-phosphate (DOXP/MEP) pathway. Acta Biochim Pol. 48(3):66372 